\title{
Weighted Immunization Strategy on Real Networks
} \author{
Jia Zhao ${ }^{1, \text { a }}$, Simeng Wang ${ }^{2}$, Zixu Zhou ${ }^{2}$, Zitong Zhou ${ }^{2}$ \\ ${ }^{1}$ Department of Information Security, Beijing University of Aeronautics and Astronautics, Beijing, \\ 100191, China \\ ${ }^{2}$ Department of Applied Mathematics, Beijing University of Aeronautics and Astronautics, Beijing, \\ 100191, China \\ aemail: jj19941031@sina.com
}

Keywords: Immunization, Community Structure, Complex Networks

\begin{abstract}
In this paper, we propose a weighted immunization strategy on real networks with community structure. We first introduce the concept of bridge nodes on networks. It may be helpful for controlling the spread of epidemic to immunize the bridge nodes in the network with community structure. Then, we present the SIR model and the classic immunization model, and add the immunization of bridge nodes to the classic immunization model. Finally, we simulate the model by using the data of real community network, finding that to combine the bridge nodes and nodes with the most degree at some ratio contributes to the immunization. The ratio can be work out.
\end{abstract}

\section{Introduction}

Complex network is an interdiscipline based on graph theory. It is characteristic of small-world, scale-free, self-organization and attractors [1]. Complex network can be used to study the real social network. Moreover, it can simulate the spread of epidemic on real social network.

Immunization has long been a problem in studying the spread of epidemic on complex networks. The classic immunization strategies can be divided into random immunization, target immunization and acquaintance immunization [2]. The random immunization is to immunize some nodes randomly. The target immunization is to immunize some special nodes, especially the nodes with large degree, usually working efficiently. The acquaintance immunization is used when the special nodes can not be picked up. It means to immunize some random nodes and their neighbor nodes [3].

In the real social network, there is always the community structure [4]. And the bridge nodes communicate the communities. Immunizing the bridge nodes will block the way where the epidemic spread from a community to another one. So, we can assume that immunizing this nodes will make much sense.

This paper adds the bridge nodes into immunization and conducts a numerical simulation experiment on real social network. Then, we can get a better weighted immunization strategy.

\section{The model}

The classic model of epidemic spread is SIR model. It divides all nodes into three states, susceptible, infective and recovered [5]. We assume that the recovered will get immunized. When the epidemic bursts, every individual will be in one of the three states. The susceptible can turn to the infective at the ratio $\beta$, and the infective can turn to the recovered at the ratio $\gamma$.

For clarity, we need to introduce the concept of degree for a given node. The degree of a node is the number of its links. If a node has a larger degree, it means that this node is more influential.

As we know, the real social network has community structure. There are always bridge nodes linking them. In order to control the epidemic, immunizing the bridge may work effectively. How to find the bridge nodes has become the first problem.

We choose an existing network, numbering all nodes and memorizing their edges. Firstly, choose a random node from all nodes. Visiting its neighbors successively in the second step [6]. 


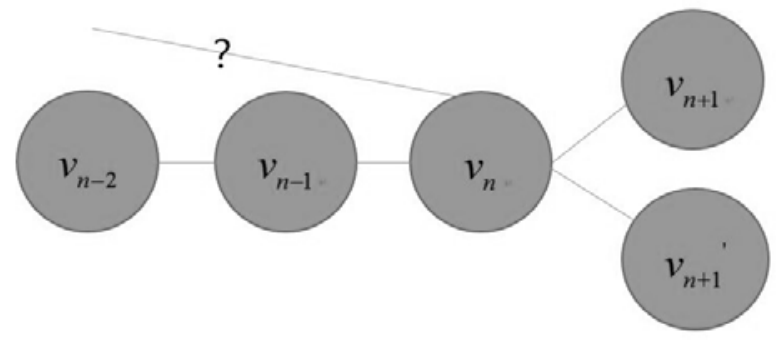

Fig.1 Find the suspicious node

For the node $v_{n}$, searching for whether there is $v_{i}(0 \leq i<n-1)$ linked to it. If it exists, continue to visit the next node $v_{n+1}$ and repeat it to $v_{n+1}$. If it does not exist, $v_{n-1}$ may be a bridge node.

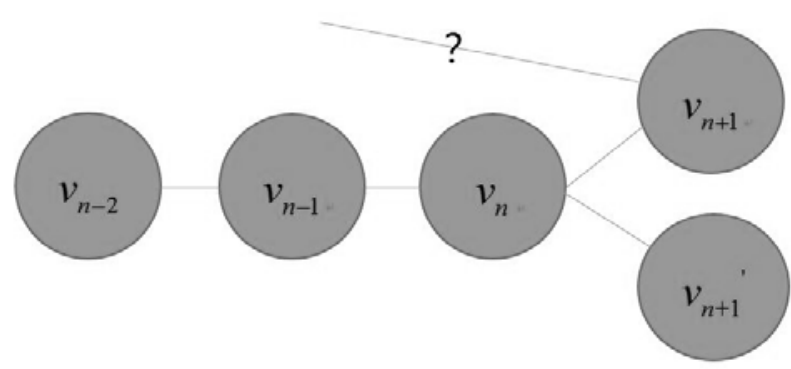

Fig.2 Find the bridge node

Next, choose two neighbors of $v_{n}, v_{n+1}$ and $v_{n+1}$. Search whether there is $v_{i}(0 \leq i \leq n-1)$ linked to $v_{n+1}$ or $v_{n+1}$ 'in the former nodes. If it exists, continue to visit the next node $v_{n+1}$ and repeat it to $v_{n+1}$. If it does not exist, $v_{n-1}$ is judged as a bridge node, memorizing its number [6].

This paper experiments on real social network. The data is provided by Stanford Network Analysis Program [7]. It is from a who-trust-whom online social network of a general consumer review site Epinions.com. In this site, members can choose whether to trust each other. All the trust relationships interact and form the Web of Trust which is then combined with review ratings to determine which reviews are shown to the user [7].

\section{Test results}

To explore the effect of bridge node, we contrast the epidemic spread in non-immunization, bridge node immunization and max-degree node immunization.

The following graph shows the different features of non-immunization, bridge node immunization and max-degree node immunization.

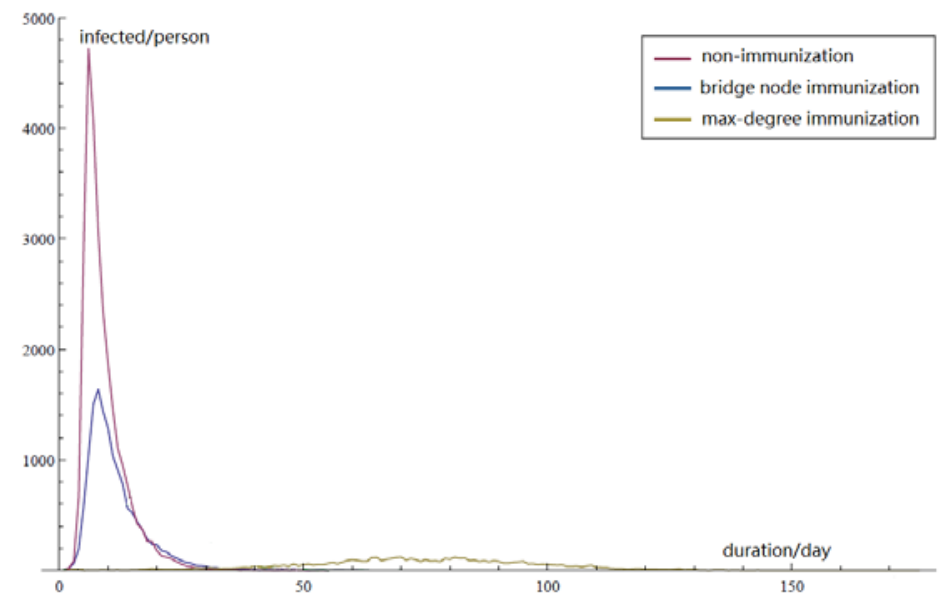

Fig.3 The infecting curve in the three kinds of immunization 
In Fig.3, the line with a sharp point stands for the case of non-immunization. It is easy to understand that if the system has no node to be vaccinated, the infected nodes will be dominated the entire network. The epidemic without immunization bursts quickly and infects quite a few individuals. And its duration is relatively short. The smooth curve lies at the bottom of the figure stands for the case of max-degree immunization. The max-degree immunization makes the infected people less than it in the others. However, the duration is much longer. It is not beneficial to control the epidemic as soon as possible. We can see from the curve that this immunization strategy performs the best. The line between them is the curve for bridge node immunization. The epidemic spread with bridge node immunization has almost the same duration as it without immunization, but the infective people is less.

We can see that the bridge node immunization has its unique merit. To make it clear, we come up with a new model, combining the max-degree immunization and the bridge node immunization, named it weighted immunization. The parameter $\alpha$ is used to represent the proportion of bridge nodes accounting for total immune nodes, named weighting coefficient.

We choose 2000 nodes as the total nodes to immunize. Relative results are shown in Fig. 4. In this figure, four lines from top to bottom stand for the case of proportion equals 1.0, 0.7, 0.4, and 0.1 , respectively.

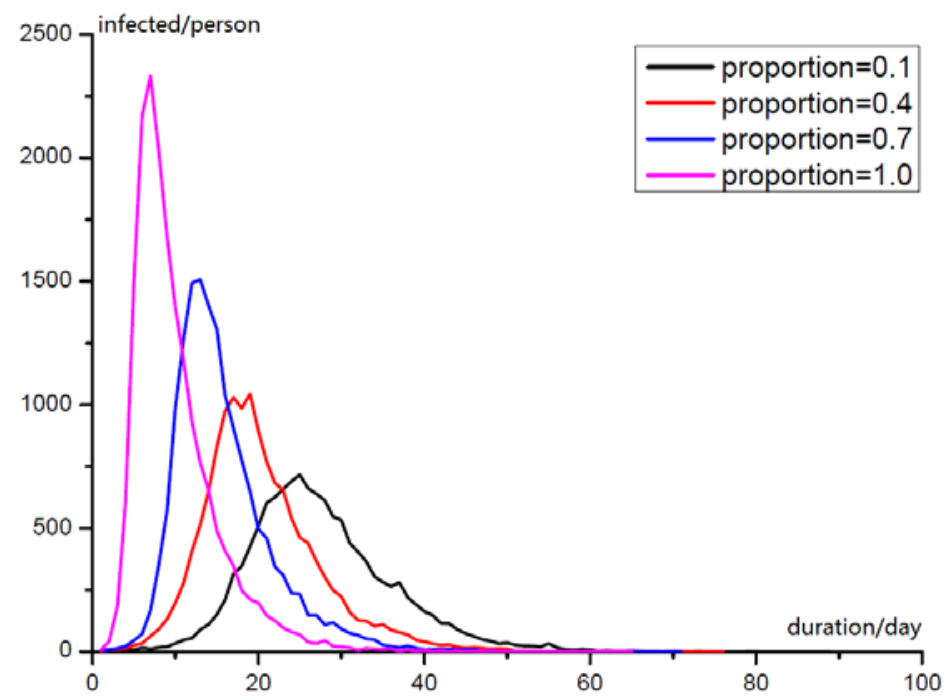

Fig.4 The infecting curve in different proportion of immune bridge nodes

The graph shows that the peak infection defers as $\alpha$ increases. But the infected number is still unclear. So, we experiment 500 times as $\alpha$ increases every 0.1. We display this result in Fig.5.

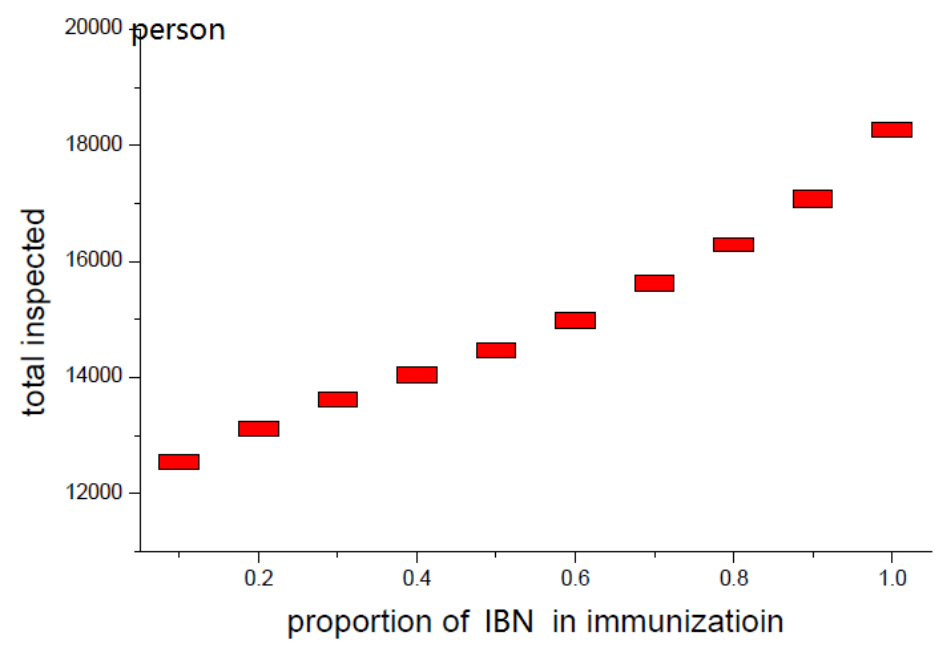

Fig.5 The total inspected in different proportion of immune bridge nodes 
The infected number and the parameter $\alpha$ have positive correlation as we can see. In this figure, the proportion of immune bridge node increases from 0 to 1 , and the total inspected nodes increases from around 12500 to19000.

Then, we experiment on studying the relationship of duration and the parameter $\alpha$. The results are shown in Fig. 6.

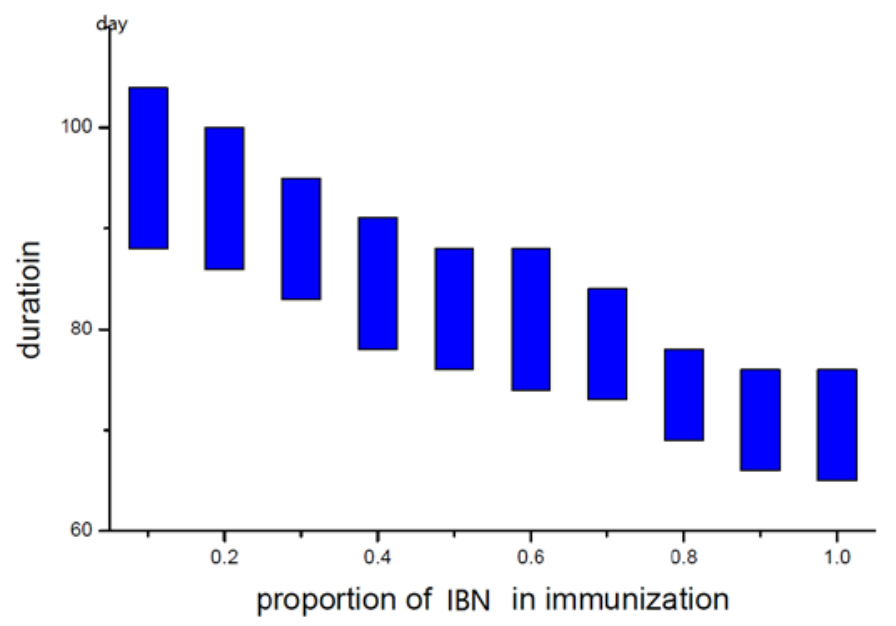

Fig. 6 The duration of different proportion of IBN in immunization

In general, as the proportion of immune bridge nodes in immunization increases, the duration of the epidemic decreases. The length of the bar indicates the fluctuation of the duration. We surprisingly notice that the duration fluctuates much at the point when $\alpha=0.6$. It means that when the proportion of immune bridge nodes in immunization equals to 0.6 , the system runs an unsteady state.

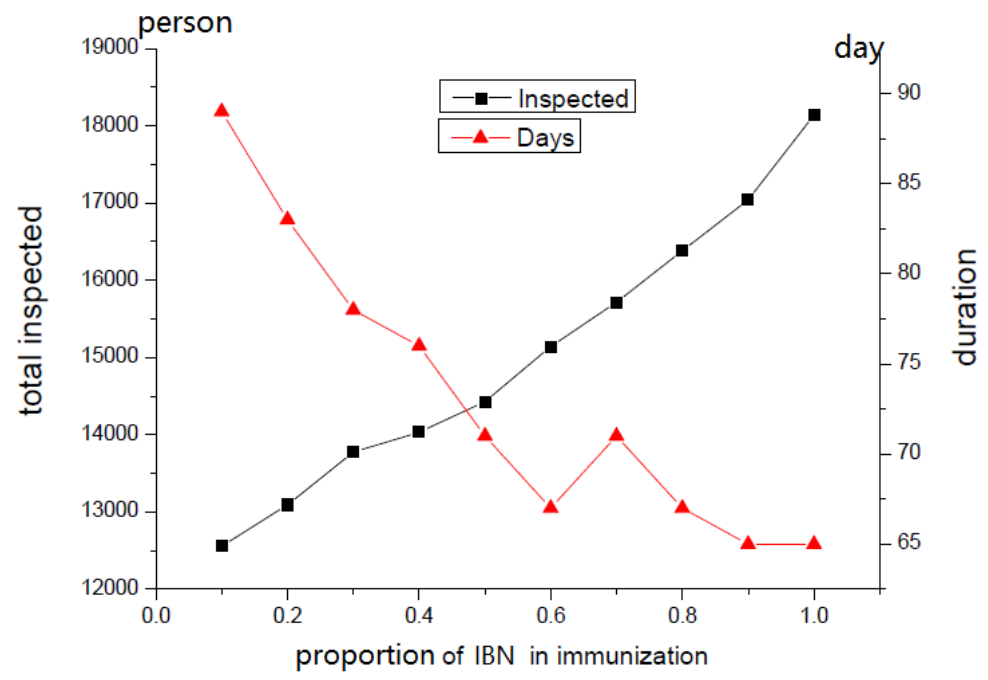

Fig.7

We take out one of the experiments as an example. As is indicated in fig.7, the curve fluctuates between $\alpha=0.6$ and $\alpha=0.8$. This phenomenon often occurs in the section. We can get the conclusion that it is an optimal solution for immunization. Besides, in this section, the total inspected number is almost two thirds of the total people. So is the duration. Considering both factors, $\alpha=0.6$ is a valuable point.

\section{Conclusion}

This paper is devoted to the merit of bridge node in social network. We try finding the best proportion of immune bridge nodes in total immunization. And we find that the infected people are relatively minor and duration is short when $\alpha=0.6$. However, it is a comprehensive result. There is not an absolutely optimal solution. 
To summarize the reasons, there may be some problems in the algorithm and the data. Firstly, the algorithm of judging bridge node can not ensure the bridge nodes completely valid because of its randomness. It may cause the lack of valid bridge nodes. Secondly, communities always overlap with each other. In this case, the bridge node has no difference with other nodes.

\section{Acknowledgement}

This work is supported by "Feng Ru Cup for BUAA Students". We really appreciate the reviewers.

\section{References}

[1] Watts D. J., Strogatz S. H. Collective dynamics of ‘small-world’networks [J], 1998.

[2] Barab’asi A L, Albert R. Emergence of scaling in random networks [J], 1999.

[3] Pastor-Satorras R., Vespignani A. Epidemic spreading in scale-free networks [J]. 2001.

[4] Marcel Salathé , James H. Jones. Dynamics and Control of Diseases in Networks with Community Structure [J], 2010.

[5] Cohen R., Havlin S., Ben-Avraham D. Efficient immunization strategies for computer networks and populations [J]. 2003.

[6] Newman M. E. J. The structure and function of complex networks [J], 2003.

[7] http://snap.stanford.edu 